BULLETIN OF THE

AMERICAN MATHEMATICAL SOCIETY

Volume 78, Number 1, January 1972

\title{
TAMING IRREGULAR SETS OF HOMEOMORPHISMS
}

\author{
BY P. F. DUVALL, JR. AND L. S. HUSCH ${ }^{1}$ \\ Communicated by O. G. Harrold, July 12, 1971
}

1. Introduction. Let $\mathscr{U}$ be an $n$-dimensional open connected manifold, $\mathscr{U}^{\infty}=\mathscr{U} \cup\{\infty\}$ the one-point compactification of $\mathscr{U}$, and $d$ a metric on $\mathscr{U}^{\infty}$. Suppose that $h$ is a homeomorphism of $\mathscr{U}$ onto itself and let $h_{\infty}$ be the extension of $h$ to $\mathscr{U}^{\infty}$. If $p \in \mathscr{U}^{\infty}$, we say that $h$ is regular at $p$ if for each $\varepsilon>0$ there is a $\delta>0$ such that $d(p, q)<\delta$ implies that $d\left(h_{\infty}^{n}(p), h_{\infty}^{n}(q)\right)<\varepsilon$ for all $n$. If $h$ is not regular at $p$, we say that $p$ is an irregular point of $h$.

Homeomorphisms with finitely or countably many irregular points have been studied extensively [4]-[10], [12]. In this paper, we consider homeomorphisms $h$ which satisfy

(1) the set of irregular points of $h$ is $P \cup\{\infty\}$, where $P$ is a $k$-dimensional continuum with $k \leqq n-2$,

and seek conditions on $h$ which imply that $P$ is nicely embedded. Details of proofs will appear elsewhere.

2. Nice homeomorphisms. Suppose that $\mathscr{U}$ and $h$ are as above. We say that $h$ is a nice homeomorphism if $h$ satisfies (1),

(2) for each $x \in \mathscr{U}-P, \varlimsup_{n \rightarrow \infty} h^{n}(x) \subset P$ and $\overline{\lim }_{n \rightarrow-\infty} h^{n}(x)=\infty$, and

(3) the mapping $f_{h}: \mathscr{U} \rightarrow P$ given by $f_{h}(x)=\lim _{n \rightarrow \infty} h^{n}(x)$ exists and is continuous.

Remarks. If $h$ satisfies (1), the work of T. Homma and S. Kinoshita [5] can be used to show that either $h$ or $h^{-1}$ satisfies (2), so that the strength of our assumptions is in (3). For example, let $h: S^{1} \times R^{2} \rightarrow S^{1} \times R^{2}$ be defined by $h(x, t)=\left(k(x), \frac{1}{2} t\right)$ where $k: S^{1} \rightarrow S^{1}$ is rotation through an irrational multiple of $\pi$ radians. Then $h$ satisfies (1) and (2) with $P=S^{1}$ $\times\{0\}$, but $h$ does not satisfy (3).

The canonical example of a nice homeomorphism is the case where $\mathscr{U}$ is an open mapping cylinder over $P$ and $h$ is a homeomorphism which "pushes in" along the product structure.

Proposition 1. If $h$ is a nice homeomorphism, then

(i) $P$ is an absolute neighborhood retract;

(ii) $f_{h}$ is onto ;

(iii) the fixed point set of $h$ is $P$;

AMS 1970 subject classifications. Primary 57E20; Secondary 57A35, 57A40.

${ }^{1}$ Research of the second author was partially supported by NSF Grant GP-15357. 
(iv) the inclusion $P \subseteq \mathscr{U}$ is a homotopy equivalence;

(v) the natural projection $p$ of $\mathscr{U}-P$ onto the orbit space $\hat{\mathscr{U}}$ of $h \mid \mathcal{U}-P$ is a covering map;

(vi) $\hat{\mathscr{U}}$ is a closed n-manifold; and

(vii) $f_{h}$ induces a map $\hat{f_{h}}: \hat{\mathscr{U}} \rightarrow P$ such that $\hat{f}_{h} p=f_{h}$.

(i)-(iv) follow from point set arguments and the fact that $h f_{h}=f_{h}$. (v)-(vii) follow from elementary facts about covering spaces and [11].

3. AFG sets and maps. If $X$ is a continuum in the ENR $M$, we say that $X$ has property $A F G$ if there is a neighborhood $W$ of $X$ in $M$ such that for each neighborhood $U$ of $X$ in $W$ there is a neighborhood $V$ of $X, V \subset U$ such that each map of $S^{1}$ into $V$ which is null homologous in $U$ is null homotopic in $U$.

It can be shown, in the spirit of [13], that the AFG property depends only on the homotopy type of $X$.

If $f$ is a proper map between manifolds, we say that $f$ is an $A F G$ map provided that $f^{-1}(x)$ has property AFG for each $x$ in the image of $f$.

4. Taming irregular sets in high dimensions. If $P$ is a polyhedron in $\mathscr{U}$, we say that $P$ is locally flat if $P$ has a triangulation in which each simplex is locally flat.

THEOREM 2. If $h$ is a nice homeomorphism with $P$ a polyhedron, $n \geqq 6$, and $k+3 \leqq n$, then $P$ is locally flat if and only if $\hat{f}_{h}$ is an AFG map.

Theorem 2 is proven by using the homotopy properties of $\hat{f}_{h}$ to show that $P$ is locally nice and by applying Bryant and Seebeck [3]. An important step in the proof is the application of L. Siebenmann's obstruction theory $[\mathbf{1 5}]$ to prove

THEOREM 3. If $\hat{f}_{h}$ is $A F G$ and $B$ is the open star of some point in $P$ in some triangulation of $P$, then $\hat{f}_{h}^{-1}(B)$ is homeomorphic to the interior of a compact manifold provided $n \geqq 6$.

5. The three-dimensional case. If $h$ is a nice homeomorphism, we say that $h$ has a cross-section if there is a closed, locally flat $(n-1)$-manifold $T \subset \mathscr{U}-P$ such that $f_{h}^{-1}(x) \cap T$ is a continuum for each $x \in P, T$ separates $\mathscr{U}$ into two components with $P$ in the bounded component, and $h(T) \cap T$ $=\varnothing$.

THEOREM 4. Let $h$ be a nice homeomorphism with cross-section, $n=3$, and $k=1$. Then $P$ is locally tame at each point and $\mathscr{U}$ is homeomorphic to the interior of a cube with $q$ handles, where $q=\operatorname{rank} H_{1}(P)$.

The proof of Theorem 4 is a lengthy argument using standard tools in three-dimensional topology. An important step in the proof involves an appeal to a taming theorem of D. R. McMillan [14]. 
If $p \in \mathscr{U}$, we say that $h$ is positively regular at $p$ if for each $\varepsilon>0$ there exists a $\delta>0$ such that $d(p, q)<\delta$ implies $d\left(h^{n}(p), h^{n}(q)\right)<\varepsilon$ for all $n>0$.

Proposition 5. If $h$ satisfies (1) and (2), $k=1, P \nsubseteq S^{1}, h \mid P=i d e n t i t y$, and $h$ is positively regular on $\mathscr{U}$, then $h$ is a nice homeomorphism.

Theorem 4, then, has an obvious restatement in terms of positive regularity. Examples can be given to show that Theorem 4 cannot be extended to higher dimensions. In fact, the construction of $\mathbf{M}$. Brown [2] using the Andrews-Curtis Theorem [1] can be used to construct, for each $n \geqq 4$ and $1 \leqq k \leqq n-3$, a homeomorphism $h$ which satisfies (1) and (2) with $\mathscr{U}=\boldsymbol{R}^{n}$ and $P$ a wildly embedded $k$-cell, such that $h$ has a cross-section and is positively regular on $\boldsymbol{R}^{n}$.

\section{REFERENCES}

1. J. J. Andrews and M. L. Curtis, n-space modulo an arc, Ann. of Math. (2) 75 (1962), 1-7. MR 25 \# 2590.

2. M. Brown, Wild cells and spheres in higher dimensions, Michigan Math. J. 14 (1967), 219-224. MR 36 \# 4533.

3. J. L. Bryant and C. L. Seebeck III, Locally nice embeddings in codimension three, Quart. J. Math. Oxford Ser. (2) 21 (1970), 265-272.

4. T. Homma and S. Kinoshita, On a topological characterization of the dilation in $E^{3}$, Osaka Math. J. 6 (1954), 135-144.

5. - On homeomorphisms which are regular except for a finite number of points, Osaka Math. J. 7 (1955), 29-38. MR 16, 1140.

6. L. S. Husch, $A$ homotopy theoretic characterization of the translation in $E^{n}$, Compositio Math. (to appear).

7. - Topological characterization of the dilation and the translation in Frechet spaces, Math. Ann. 190 (1970), 1-5.

8. , Topological characterization of the dilation in $E^{n}$, Proc. Amer. Math. Soc. 28 (1971), 234-236.

9. S. K. Kaul, On almost regular homeomorphisms, Canad. J. Math. 20 (1968), 1-6. MR 36 \# 5908.

10. B. v. Kerékjártó, Topologische Characterisierungen der linearen Abbildungen, Acta Litt. Acad. Sci. Szeged 6 (1934), 235-262.

11. S. Kinoshita, Notes on covering transformation groups, Proc. Amer. Math. Soc. 19 (1968), 421-424. MR 36 \# 5921.

12. - On quasi-translations in 3-space, Fund. Math. 56 (1964), 69-79. MR 30 \#1502.

13. R. C. Lacher, Cell-like spaces, Proc. Amer. Math. Soc. 20 (1969), 598-602. MR 38 \#2754.

14. D. R. McMillan, Jr., Local properties of the embedding of a graph in a three-manifold, Canad. J. Math. 18 (1966), 517-528. MR 34 \#6754.

15. L. C. Siebenmann, The obstruction to finding a boundary for an open manifold of dimension greater than five, Thesis, Princeton University, Princeton, N.J., 1965.

Department of Mathematics, Virginia Polytechnic Institute And State UniVERSITY, BLACKSBURG,'VIRGINIA 24061

Current address: (P. F. Duvall, Jr.) Department of Mathematics and Statistics, Oklahoma State University, Stillwater, Oklahoma 74074

Current address: (L. S. Husch) Department of Mathematics, University of Tennessee, Knoxville, Tennessee 37916 